\title{
Macrophage migration inhibitory factor promotes eosinophil accumulation and tissue remodeling in eosinophilic esophagitis
}

\author{
HS de Souza ${ }^{1,2}$, CA Tortori ${ }^{1}$, L Lintomen ${ }^{3}$, RT Figueiredo ${ }^{4}, \mathrm{C}_{\text {Bernardazzi }}{ }^{1}$, L Leng $^{5}$, R Bucala $^{5}$, K Madi $^{1}$, \\ F Buongusto $^{1}$, CCS Elia ${ }^{1,2}$, MTL Castelo-Branco $^{6}$ and MT Bozza ${ }^{3}$
}

Macrophage migration inhibitory factor (MIF) is involved in eosinophil biology and in type 2 inflammation, contributing to allergic and helminthic diseases. We hypothesized that MIF participates in the pathogenesis of eosinophilic esophagitis (EoE), an allergic condition characterized by esophageal eosinophilic inflammation. MIF is highly expressed in esophageal mucosa of patients with EoE, compared with gastro-esophageal reflux disease and control patients, where it co-localizes predominantly with eosinophils. In vitro, recombinant MIF promotes human eosinophil chemotaxis, while MIF antagonist and CXCR4 antagonist, AMD3100, revert this effect. In a model of EoE induced by ovalbumin, Mif-deficient mice have reduced inflammation and collagen deposition compared with wild-type (WT) mice. Importantly, treatment of WT mice with anti-MIF or with AMD3100 during the challenge phase prevents accumulation of eosinophils and tissue remodeling. Conversely, recombinant MIF promoted tissue eosinophil inflammation in allergic mice. Together, these results implicate MIF in the pathogenesis of esophageal inflammation and suggest that targeting MIF might represent a novel therapy for EoE.

\section{INTRODUCTION}

Eosinophilic esophagitis (EoE) constitutes an emerging clinicopathologic condition with symptoms mimicking gastroesophageal reflux disease (GERD) and an aberrant infiltration of eosinophils into the esophageal mucosa. ${ }^{1,2}$ Although EoE may be clinically similar to GERD, the acid suppression therapy is usually ineffective and most patients have a high rate of association with allergic disorders. ${ }^{3}$ In addition, some distinctive pathologic characteristics have been reported in esophageal biopsy specimens of patients with $\mathrm{EoE}^{4}$ and in experimental models of EoE, ${ }^{5,6}$ including epithelial cell hyperplasia and subepithelial fibrosis. In regard to the pathogenesis of EoE, evidence supports a role for a predominant $\mathrm{Th} 2$ type of immune response with an overproduction of cytokines such as inter- leukin (IL)-5 and IL-13, ${ }^{7,8}$ and a critical role for the eosinophil chemoattractant and activator eotaxin-3, produced by esophageal epithelial cells. ${ }^{9}$

Macrophage migration inhibitory factor (MIF) is a proinflammatory cytokine that participates in the immune response to several infectious agents but also has a prominent role promoting tissue damage in infectious and sterile inflammatory conditions. ${ }^{10-12}$ Accumulating evidence demonstrates that MIF is important in Th2 type immune responses. Activated Th2 cells have increased MIF mRNA and protein, while eosinophils have the pre-formed protein and are able to secrete high quantities of MIF upon stimulation. ${ }^{13,14}$ MIF also profoundly affects eosinophil physiology promoting eosinophil differentiation, activation, migration and survival. ${ }^{15-17}$

\footnotetext{
${ }^{1}$ Serviço de Gastroenterologia \& Laboratório Multidisciplinar de Pesquisa, Department of Internal Medicine, Hospital Universitário, Universidade Federal do Rio de Janeiro, Rio de Janeiro, Brazil. ${ }^{2}$ D'Or Institute for Research and Education (IDOR), Rio de Janeiro, Brazil. ${ }^{3}$ Laboratório de Inflamação e Imunidade, Departamento de Imunologia, Instituto de Microbiologia, Universidade Federal do Rio de Janeiro, Rio de Janeiro, Brazil. ${ }^{4}$ Instituto de Ciências Biomédicas, Pólo de Xerém, Universidade Federal do Rio de Janeiro, Rio de Janeiro, Brazil. ${ }^{5}$ Department of Medicine/Rheumatology, Yale University School of Medicine, The Anlyan Center, New Haven, CT, USA and ${ }^{6}$ Laboratório de Imunologia Celular, Instituto de Ciências Biomédicas, Universidade Federal do Rio de Janeiro, Rio de Janeiro, Brazil. Correspondence: HSP de Souza (hsouza@hucff.ufrj.br or heitor.souza@gmail.com)
}

Received 20 May 2014; accepted 2 January 2015; published online 25 February 2015. doi:10.1038/mi.2015.6 
In mouse models of asthma and allergic rhinitis the lack of MIF causes an almost complete abrogation of the cardinal signs of the disease including mucus secretion, eosinophilic inflammation and airway hyper-responsiveness. ${ }^{15,18,19}$ We observed that $\mathrm{Mif}^{-/-}$mice have reduced amounts of IL-13 and IL-5 in the lungs but similar amounts of IL- 4 and IL-13 in the lymph nodes or serum IgE upon ovalbumin (OVA)-induced allergic inflammation. ${ }^{15}$ Moreover, MIF has been shown to participate in the response to helminthic infection as demonstrated by the inefficient control of the adult forms of these parasites or the reduced inflammation and tissue damage in the absence of MIF. ${ }^{16,20,21}$ Atopic patients have increased amounts of MIF in the bronchoalveolar lavage fluids, sputum, and sera. ${ }^{13,22}$

Because MIF appears to be an important component of type 2 immune responses, we aimed to uncover a potential involvement of MIF in the pathogenesis of EoE. We found that MIF was increased in the esophagus of patients diagnosed with EoE, including in infiltrating eosinophils, macrophages, and lymphocytes. Human eosinophils migrated in response to MIF, an effect reverted by a small molecule antagonist of MIF and by an antagonist of CXCR4. In a mouse model of allergic EoE induced by OVA sensitization and challenge, genetic Mif deficiency prevented the eosinophil accumulation and collagen fiber deposition. Finally, MIF blockage in the challenge phase suggests that MIF might be a possible target for treating EoE.

\section{RESULTS}

\section{Expression of MIF is increased in the EoE esophageal} mucosa

Mucosal samples were studied in regard to MIF expression at both the protein and mRNA levels. MIF-positive cells revealed by immunohistochemistry were distributed throughout the epithelial layer, and a marked increase was observed in the mucosa of EoE compared with GERD and control patients (Figure 1a). Under higher magnification, MIF-positive cells were shown to be predominantly eosinophils, with a clear staining within cytosolic granules and to a lesser extent in the extracellular milieu (Figure 1b). Quantitative analysis demonstrated that MIF-positive cells were significantly increased in mucosal samples from EoE compared with GERD, and control patients, and also in GERD compared with controls (Figure 1c). The esophageal mucosa also showed a characteristic distribution of MIF-positive cells, in great part corresponding to eosinophils (Figure $\mathbf{1 b}$ ). In fact, we found a positive correlation between MIF-positive cells and eosinophils in EoE patients, and the Spearman's rank correlation coefficient calculated between MIF-positive cells and eosinophils was $r=0.863 ; P=0.001$. Moreover, MIF-positive cells were also positively correlated with the male gender $(r=0.313$; $P=0.032$ ), but not with other clinical or laboratory data. In addition, MIF mRNA levels were significantly increased in mucosal samples from EoE compared with GERD, and control patients, and also in GERD compared with controls (Figure 1d). Esophageal explants from EoE, GERD, and control patients were cultured under different conditions, and supernatants were harvested for measurement of MIF concentrations by enzyme-linked immunosorbent assay. The basal concentrations of MIF were significantly higher in EoE compared with GERD and control supernatants. However, after exposure to lipopolysaccharide or phorbol myristate acetate, the amounts of MIF did not change significantly in any of the patient groups (Figure 1e). These results indicate that MIF mRNA and protein are highly increased in the esophagus of EoE patients.

\section{Eosinophils are the predominant MIF-positive immune cells in the EoE esophageal mucosa}

Immunofluorescence images revealed MIF expression mostly at the superficial epithelium of EoE tissue samples, within the inflammatory/immune cell infiltrate. To assess which inflammatory cells co-express MIF, sections were incubated with a combination of anti-CD3, anti-CD68, or anti-major basic protein, with anti-MIF. Double-positive cells were counted in relation to the most representative inflammatory/immune cells of the esophageal mucosa. Co-localization of MIF (red) with $\mathrm{T}$ cells (CD3), macrophages (CD68), and eosinophils (MBP) (green) was performed by double immunofluorescence with confocal microscopy analysis. In the inflamed EoE mucosa, MIF co-localizes predominantly with eosinophils (median $42 \%$ ) and to a lesser extent with macrophages (median 26\%) and T cells (median 19\%). On the other hand, in inflamed GERD mucosa MIF co-localizes more with macrophages (median 20\%) and $\mathrm{T}$ cells (median 16\%), and less with eosinophils (median 11\%) (Figures 2a and b). These data indicate that eosinophils are the major source of MIF in the esophageal mucosa of EoE.

\section{Effect of MIF blockade on other cytokines in esophageal explants}

Next, we analyzed whether MIF could modulate the production of other cytokines, including the ones characteristically associated with EoE. As expected, the basal concentrations of eotaxin-3, IL-13, and IL-5 were significantly higher in supernatants from EoE compared with GERD and control patients (Supplementary Figures 1A-C online). In contrast, concentrations of IL- $1 \beta$ were significantly higher in GERD, compared with EoE and control supernatants (Supplementary Figure 1D). However, treating explants with different concentrations of neutralizing anti-MIF antibody did not result in any significant change in the amounts of the aforementioned cytokines in any of the patient groups.

\section{MIF induces eosinophil chemotaxis}

In another set of experiments, we investigated the ability of MIF to induce human eosinophil chemotaxis in a transwell system. A remarkable response was observed upon the treatment with MIF, significantly increasing the attraction of eosinophils at $1 \mathrm{ng} \mathrm{ml}^{-1}$. Interestingly, the effect of MIF was similar to the one observed with the well-established eosinophil chemo-attractant eotaxin (Figure 3a). Previous studies demonstrated that MIF binds to and activates the CXCR4 receptor inducing leukocyte recruitment and inflammation. ${ }^{23-25}$ The chemotactic effect of 
a

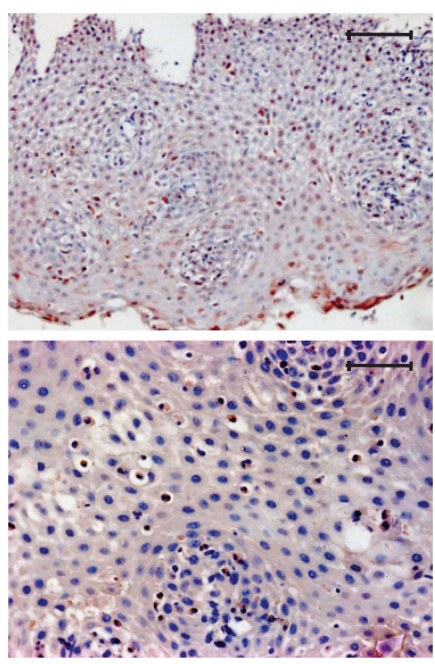

GERD
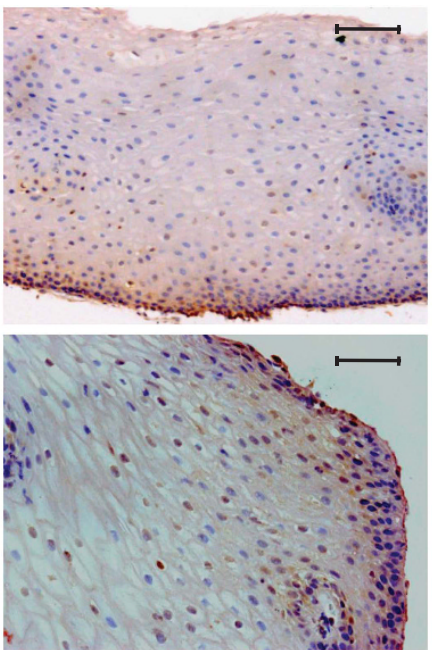

Control

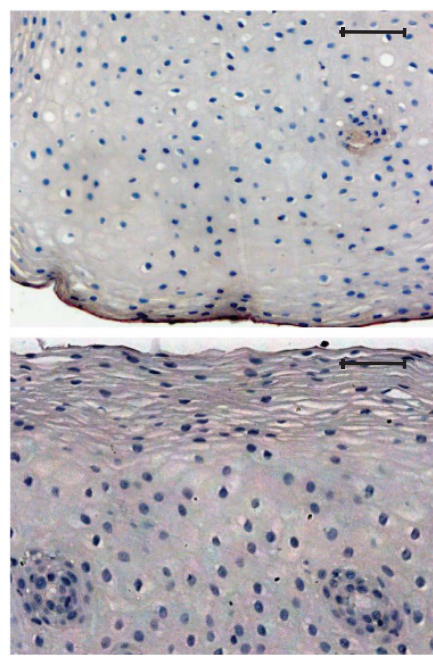

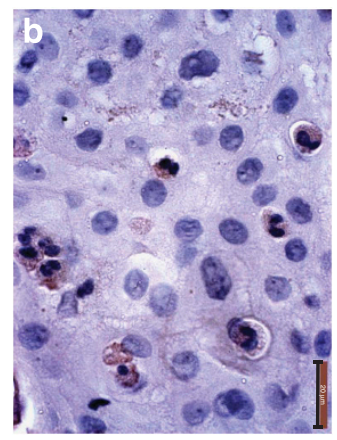

C

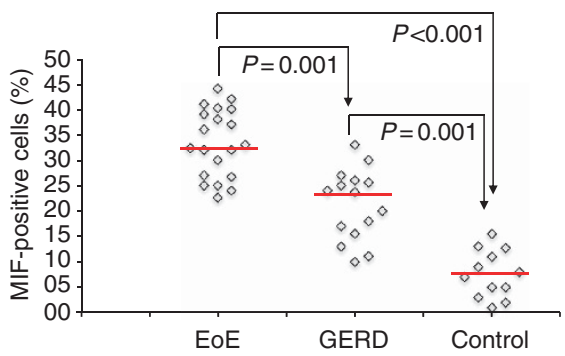

d 1.8

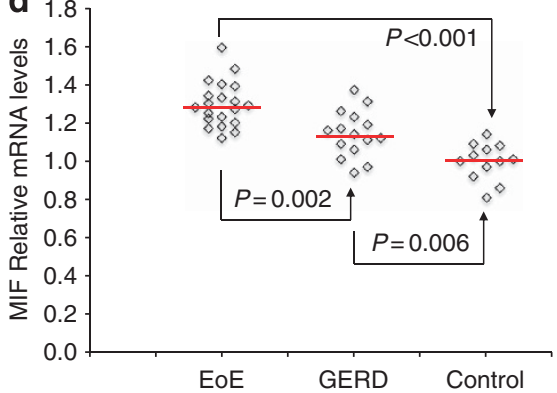

e

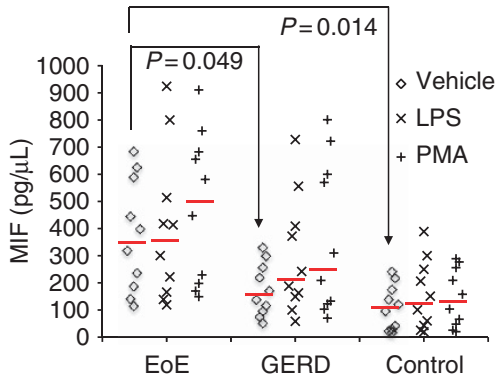

Figure 1 Expression and modulation of macrophage migration inhibitory factor (MIF) protein and mRNA in eosinophilic esophagitis (EoE). Mucosal samples were obtained by endoscopy at inflamed areas of the esophagus, and studied by immunohistochemistry. MIF was characteristically expressed at the epithelium, and a marked increase was observed in the mucosa of EoE compared with gastro-esophageal reflux disease (GERD) and control patients (a, c). Using oil-immersion technique, most MIF-positive cells were morphologically compatible with eosinophils, while cytosolic granules and extracellular staining for MIF could also be detected. Length bars represent $20 \mu \mathrm{m}$ (b). Mifgene expression in the esophageal mucosa was determined by RT-qPCR. Levels of MIF mRNA were normalized to the RPL32 and GAPDH RNA genes (d). MIF concentrations with or without the addition of phorbol myristate acetate (PMA) $\left(1 \mu \mathrm{g} \mathrm{ml}^{-1}\right)$, and lipopolysaccharide (LPS) $\left(1 \mu \mathrm{g} \mathrm{ml}^{-1}\right)$ (e) are expressed as picogram of cytokine per milliliter of culture supernatant and normalized by protein contents. Significant changes in relation to the control group are highlighted. Values presented as percentages of MIF-positive cells, MIF mRNA relative levels, and MIF concentrations are individually represented, and horizontal bars represent medians of $12-20$ samples in each group. Differences were analyzed using ANOVA on ranks with Dunnett's test.

MIF on eosinophils was further tested by the addition of ISO-1, an MIF antagonist, and AMD3100, a CXCR4 antagonist, to cell culture medium in the upper chambers. Both ISO-1 and AMD3100 significantly inhibited the chemotactic effect of MIF (Figure 3b).

\section{Genetic deficiency of MIF prevented eosinophilic infiltration and collagen deposition in mice esophagus}

To characterize the putative role of MIF in a mouse model of EoE, groups of wild-type (WT) and $\mathrm{Mif}^{-1-}$ mice in the C57BL/6 background were sensitized and challenged with OVA. WT mice sensitized and challenged with OVA exhibited a marked eosinophil recruitment to the esophagus compared with WT controls while $\mathrm{Mif}^{-/-}$mice were protected against the increased infiltration of eosinophils (Figures $4 \mathbf{a}-\mathbf{c}$ ). Esophagus of WT mice, but not of $\mathrm{Mif}^{-/-}$mice, presented a significant increase in collagen deposition upon OVA challenge (Figures $\mathbf{4 a}$ and $\mathbf{d}$ ). In fact, the eosinophil numbers and density of collagen fibers in the esophageal tissue of $\mathrm{Mif}^{-/-}$ mice sensitized and challenged with OVA were comparable to that noted in saline control mice.

To further characterize the experimental EoE and the induction of MIF in the model, we performed indirect immunoperoxidase experiments, which showed a strong reactivity in WT mice sensitized and challenged with OVA, compared with controls and with $\mathrm{Mif}^{-/-}$mice (Figures 5a and $\mathbf{b}$ ). In addition, we performed real-time PCR assessments of cytokine-related genes, including MIF, eotaxin, IL-5, IL-13, and TSLP (thymic stromal lymphopoietin), a cytokine recently shown to contribute to the pathogenesis of EoE. ${ }^{26}$ In contrast to results in human EoE tissue, and to MIF protein abundance in the experimental model, the messenger RNA for MIF was not 
a
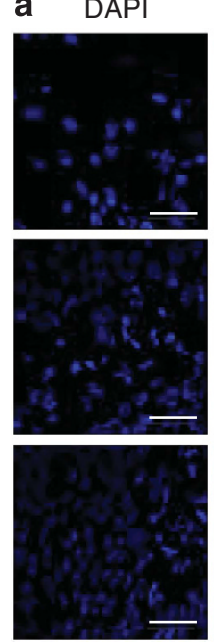

MIF
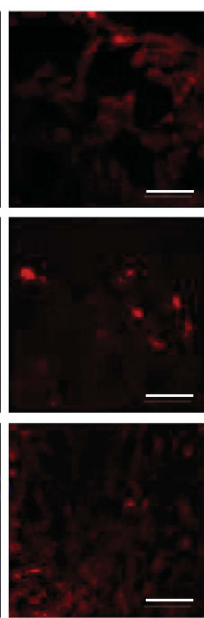

Overlay
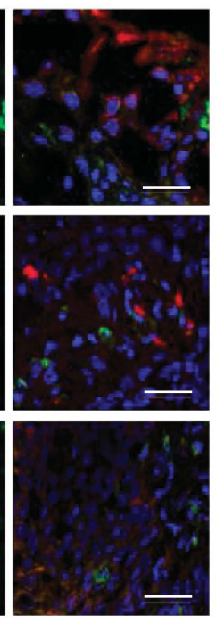

DAPI
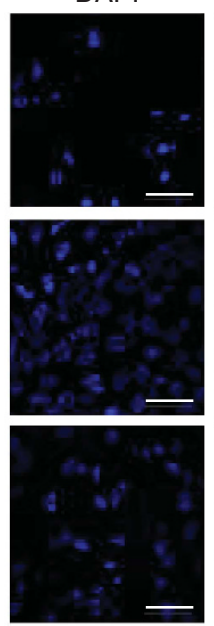

MIF
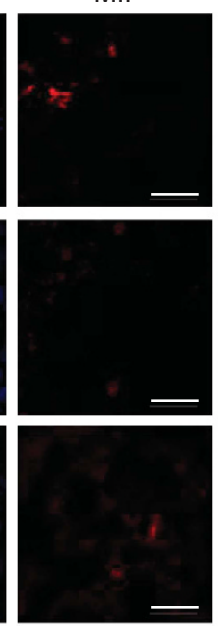

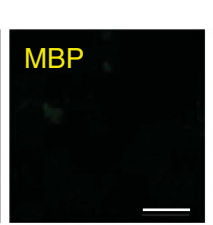

CD68

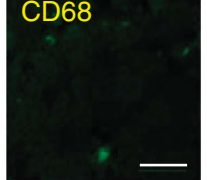

CD3

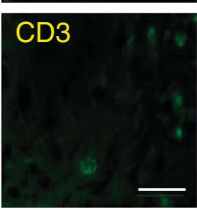

EoE

GERD

\section{Overlay}
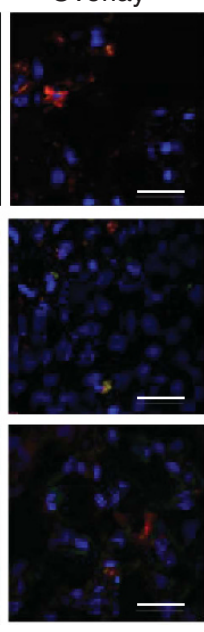

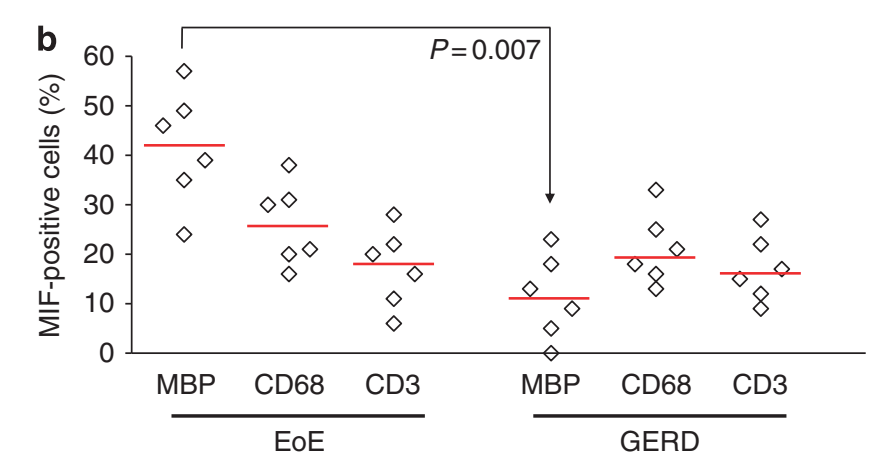

Figure 2 Co-localization of macrophage migration inhibitory factor (MIF) with inflammatory cells in eosinophilic esophagitis (EoE). Co-localization of MIF (red) with Eosinophils major basic protein (MBP), macrophages (CD68), and T cells (CD3) (green) was performed by double immunofluorescence with confocal microscopy analysis. In the esophageal mucosa of patients with eosinophilic EoE, MIF co-localizes predominantly with eosinophils, and to a lesser extent with macrophages, and T cells. In the mucosa from gastro-esophageal reflux disease (GERD), MIF co-localizes predominantly with macrophages and $T$ cells, and significantly less with eosinophils (a). Figure representative of mucosal samples from six EoE and six GERD patients. Length bars represent $50 \mu \mathrm{m}$. Percentages of double-positive cells in the esophageal mucosa are individually represented. Horizontal bars represent medians (b).
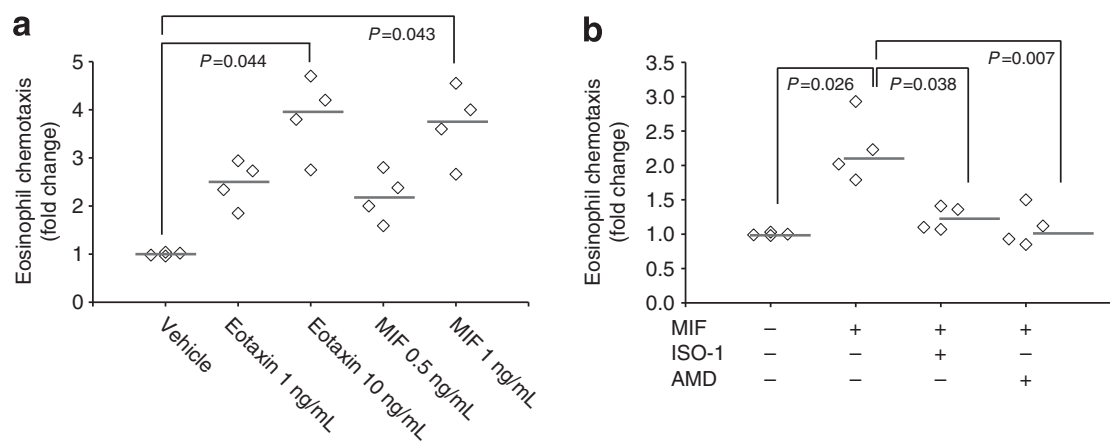

Figure 3 Eosinophil chemotaxis induced upon exposure to macrophage migration inhibitory factor (MIF). Purified peripheral blood eosinophils were incubated in the upper chambers of a $5-\mu \mathrm{m}$-pore-size polycarbonate filter in a transwell system, with cell culture medium treated with MIF (0.5 and $1 \mathrm{ng} \mathrm{ml}^{-1}$ ) or eotaxin $\left(1\right.$ and $10 \mathrm{ng} \mathrm{ml}^{-1}$ ) in the lower chambers (a). The chemotactic effect of MIF on eosinophils was further tested by the addition of MIF antagonists ISO-1 (100 $\mu \mathrm{M})$ and AMD3100 (AMD) $\left(100 \mathrm{ng} \mathrm{ml}^{-1}\right)$ to the upper chambers (b). Cell culture media without any treatment were used as negative controls and values were arbitrarily normalized to 1 . Significant changes in relation to the control group are highlighted. Horizontal bars represent medians. Differences were analyzed using ANOVA on ranks with Dunnett's test.

significantly increased in the OVA-induced animals. Interestingly, in induced animals the expression of IL-5 and Tslp did not change significantly, and eotaxin was undetectable.
In regard to IL-13 mRNA, the expression was increased in induced animals, but decreased in $\mathrm{Mif}^{-/-}$mice (Supplementary Figure 2). 

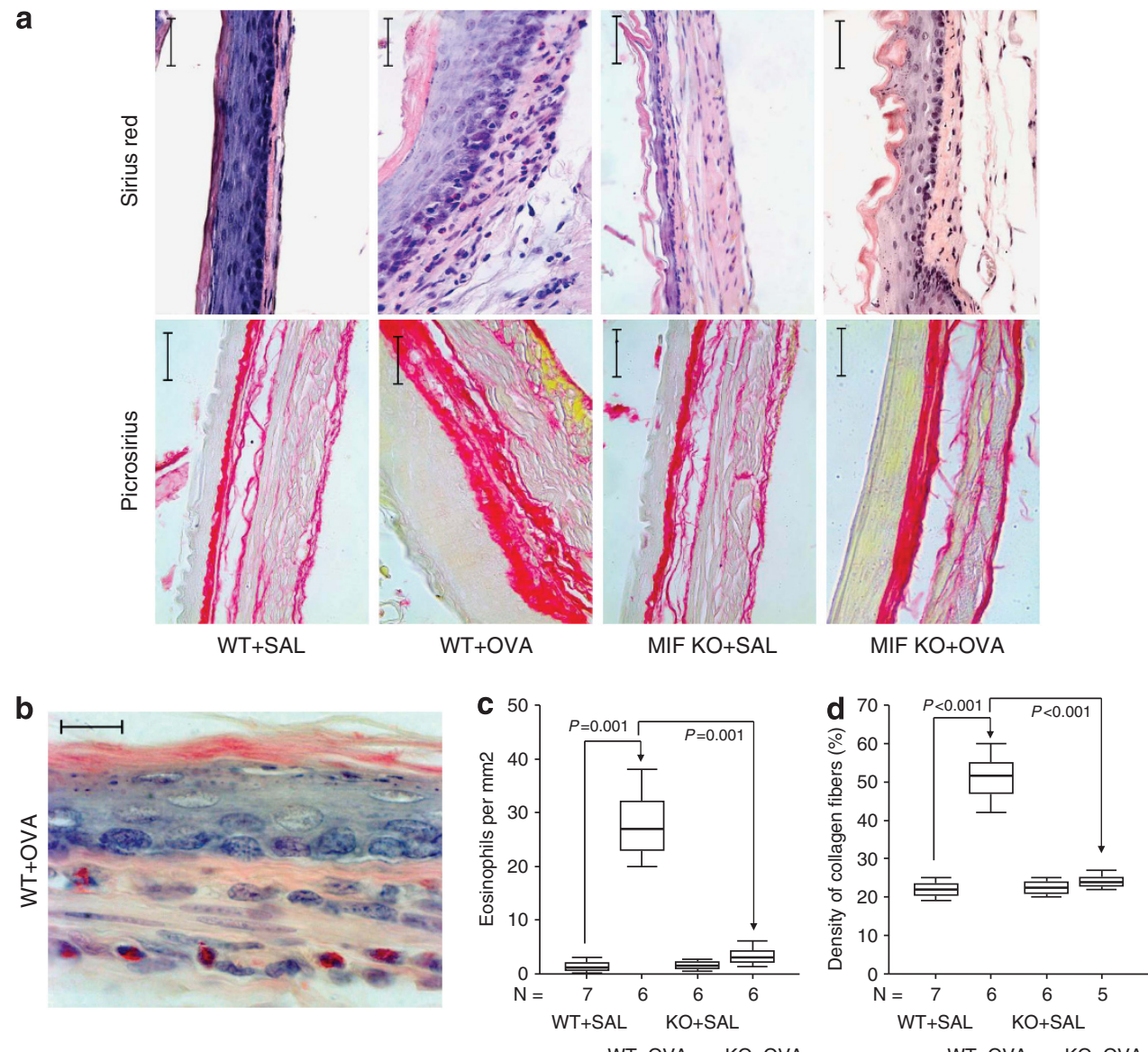

Figure $4 \mathrm{Mif}^{-/-}$animals do not develop full OVA-induced eosinophilic esophagitis. After ovalbumin (OVA) induction, Mif ${ }^{+\prime+}$ (wild type, WT + OVA) presented significant morphological changes of Sirius red- and Picrosirius-stained esophageal sections characterized by intense cellular infiltration, predominantly of eosinophils and increased wall thickness, compared with saline-treated $\mathrm{Mif}^{+/+}$mice (WT $\left.+\mathrm{SAL}\right)$, and both OVA- and saline-treated Mif $^{-1-}$ animals (KO + SAL and KO + OVA) (a). Cellular infiltration with eosinophils is shown by Sirius red staining in detail (b). Analysis of esophageal

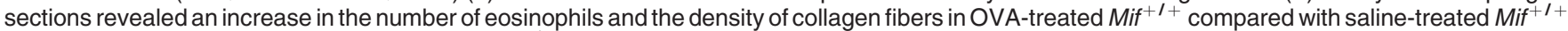
animals, and with both OVA- or saline-treated $\mathrm{Mif}^{-1-}$ animals (c, d). Horizontal bars represent medians, boxes represent the 25th and 75th percentiles, and vertical bars represent ranges of six animals per group. Length bars represent $20 \mu \mathrm{m}$. Differences were analyzed using ANOVA on ranks with Dunnett's test. MIF, macrophage migration inhibitory factor.

\section{Blockage of MIF protected against the signs of EoE in mouse model}

The lack of inflammation and tissue remodeling observed in mice deficient of Mif gene prompted us to analyze the effect of blocking MIF protein in the model of EoE. We used two strategies to block MIF effects: an anti-MIF monoclonal antibody and an antagonist of CXCR4, AMD 3100. BALB/c mice were sensitized with OVA and treated or not with antiMIF, IgG (isotype control) or AMD 3100 before each intranasal challenge. In these experiments treatment started after the sensitization phase. Treatment with anti-MIF significantly decreased the eosinophil infiltration when compared with OVA challenged group (Figures 6a and b). The IgG isotype control, as expected, did not influence the inflammation profile and was comparable to the OVA group. The analysis of tissue remodeling demonstrated that treatment with anti-MIF or AMD 3100 also prevented the deposition of collagen fibers (Figures $\mathbf{6 c}$ and d). Similarly, treatment with anti-MIF abrogated the eosinophil infiltration and the deposition of collagen fibers, compared with the OVA challenged group treated with IgG isotype control in C57BL/6 mice (Supplementary Figures $\mathbf{3 A}$ and $\mathbf{B}$ ). Collectively, these results indicate that genetic deficiency of Mif, its neutralization, or the blockage of CXCR4 receptor have a major impact on the development of mouse EoE.

\section{Recombinant MIF induces signs of EoE in OVA-immunized mice}

In another set of experiments, we sought to investigate the potential effect of MIF before the full OVA-induction of EoE. For this purpose, C57BL/6 mice were immunized with OVA, and then treated with saline, recombinant MIF, OVAinduction, both MIF and OVA-induction. Except for the saline-treated, all other OVA-immunized mice presented the typical morphological changes of Sirius red- and Picrosiriusstained esophageal sections characterized by intense cellular infiltration, predominantly of eosinophils and increased wall thickness (Figure 7a). Interestingly, our findings show that 


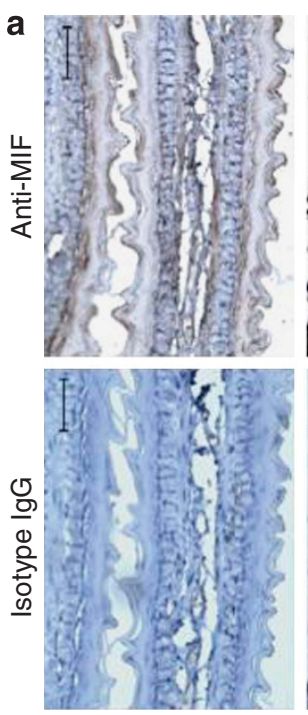

WT+SAL
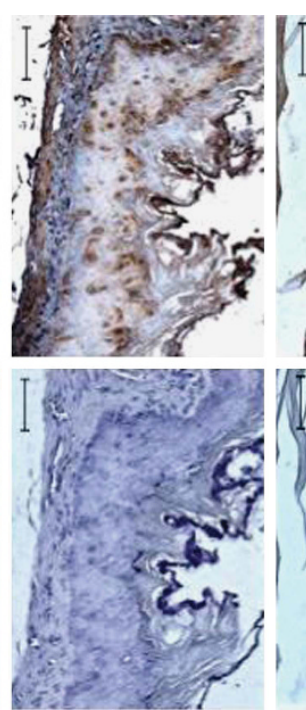

WT+OVA

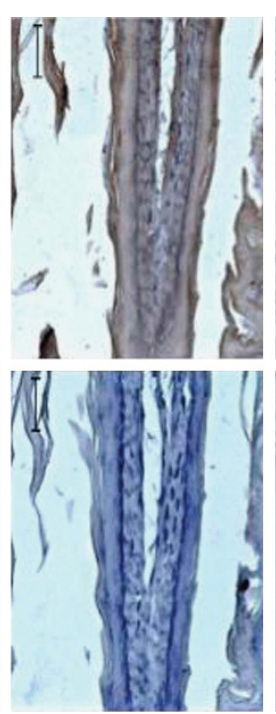

MIF KO+SAL
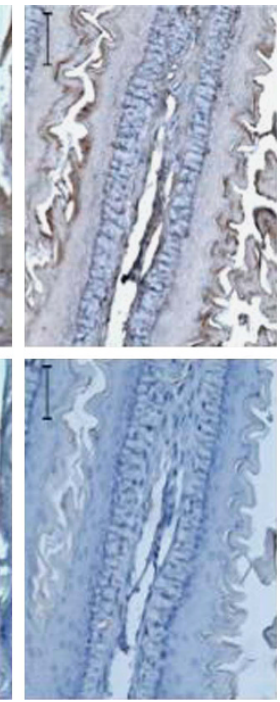

MIF KO+OVA

b

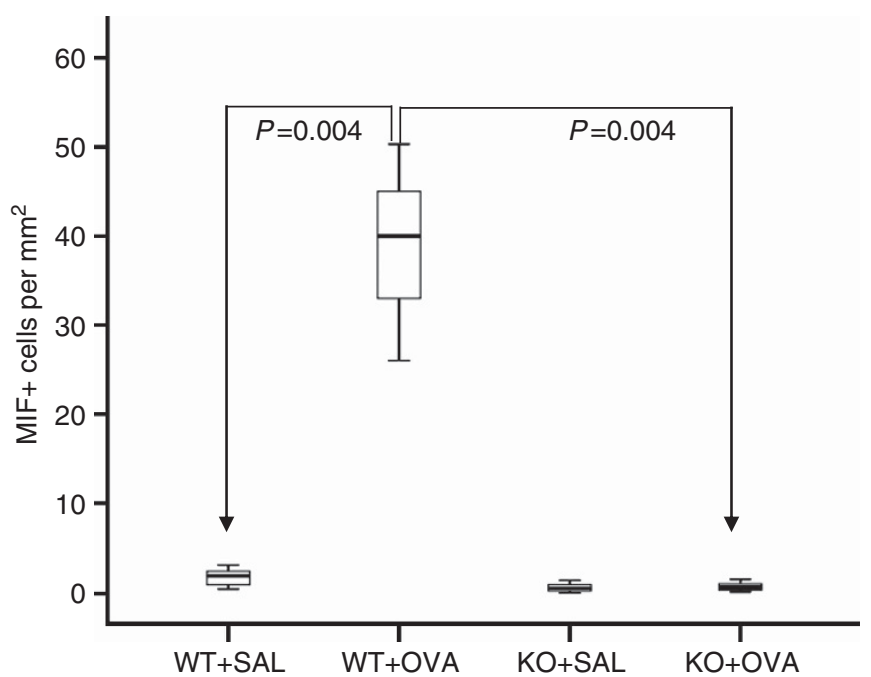

Figure 5 Macrophage migration inhibitory factor (MIF) is overexpressed in the murine model of eosinophilic esophagitis. Esophageal tissue of the experimental animals was studied by indirect immunoperoxidase. After ovalbumin (OVA) induction, Mif ${ }^{+1+}$ (wild type, WT + OVA) mice presented a significant increase in MIF-positive cells in the esophageal mucosa compared with saline-treated Mif ${ }^{++}$mice (WT + SAL), and both OVA- and salinetreated $\mathrm{Mif}^{-1-}$ animals $(\mathrm{KO}+\mathrm{SAL}$ and KO $+\mathrm{OVA})(\mathbf{a}, \mathbf{b})$. Horizontal bars represent medians, boxes represent the 25 th and 75 th percentiles, and vertical bars represent ranges of six animals per group. Length bars represent $20 \mu \mathrm{m}$. Differences were analyzed using ANOVA on ranks with Dunnett's test.

recombinant MIF is also capable of inducing eosinophilic inflammation in the esophagus of animals previously immunized with OVA (Figures $\mathbf{7 b}$ and $\mathbf{c}$ ).

\section{DISCUSSION}

We show here that MIF expression is increased in the esophagus of patients with EoE and MIF decisively participates in the inflammatory response and tissue remodeling in a mouse model of the disease. We confirm and extend the pivotal role of MIF in type 2 immune responses and in eosinophil biology. In patients with EoE, eosinophils are important source of MIF that, in turn, has the ability to directly promote their chemotaxis in vitro in a mechanism dependent on CXCR4. WT mouse treated with anti-MIF antibody or with CXCR4 antagonist and $\mathrm{Mif}^{-/-}$mouse are protected from the eosinophilic inflammation and tissue remodeling in a model of EoE induced by OVA.

Although esophageal eosinophilia has been considered the most typical characteristic of human EoE, the mechanisms involved in cell accumulation in the tissue, and the exact role of eosinophils in disease pathogenesis are yet to be determined. Nevertheless, environmental agents including food and aeroallergens have been implicated in EoE induction, suggesting a role for the type 2 immune response. ${ }^{27}$ In accordance with previous findings from other series, most of our EoE patients were male and had a history of allergy. ${ }^{28}$ In regard to tissue characteristics, we also found an increased deposition of sub-epithelial collagen fibers, in parallel with thickening of the basal cell layer, as a result of increased epithelial cell proliferation. ${ }^{29,30}$ 

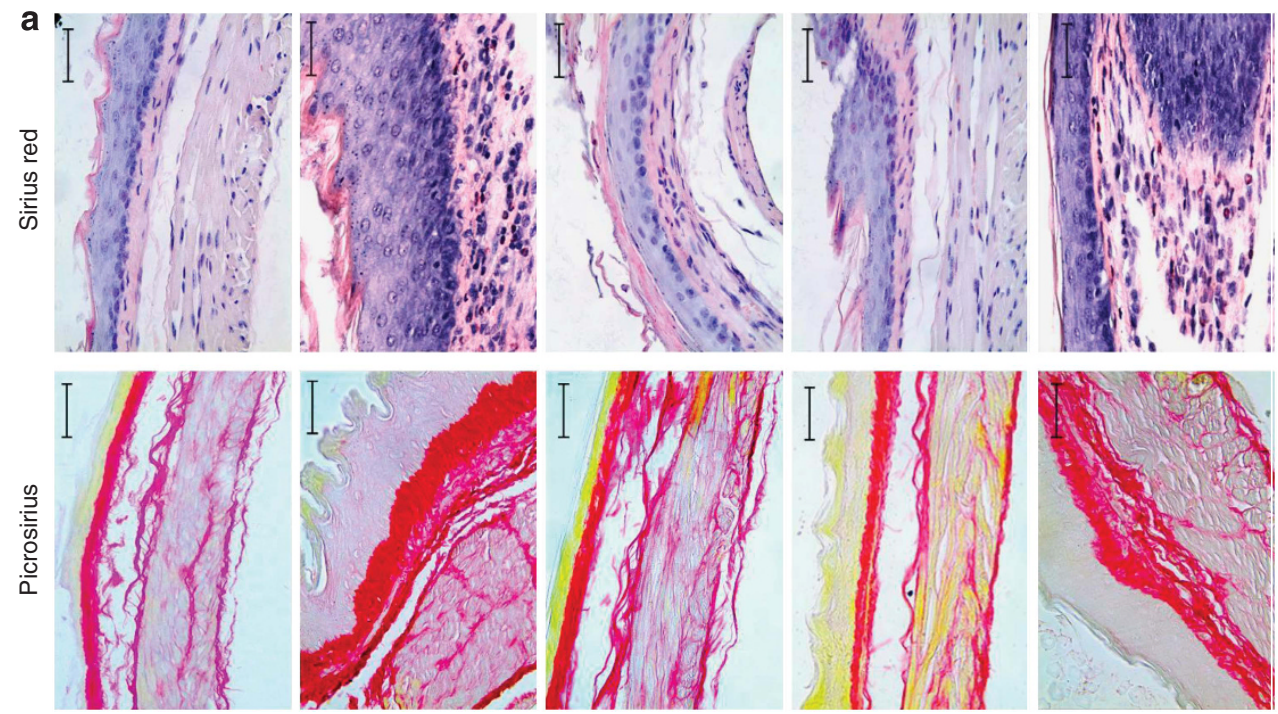

SAL

OVA

OVA+AMD

OVA+anti-MIF
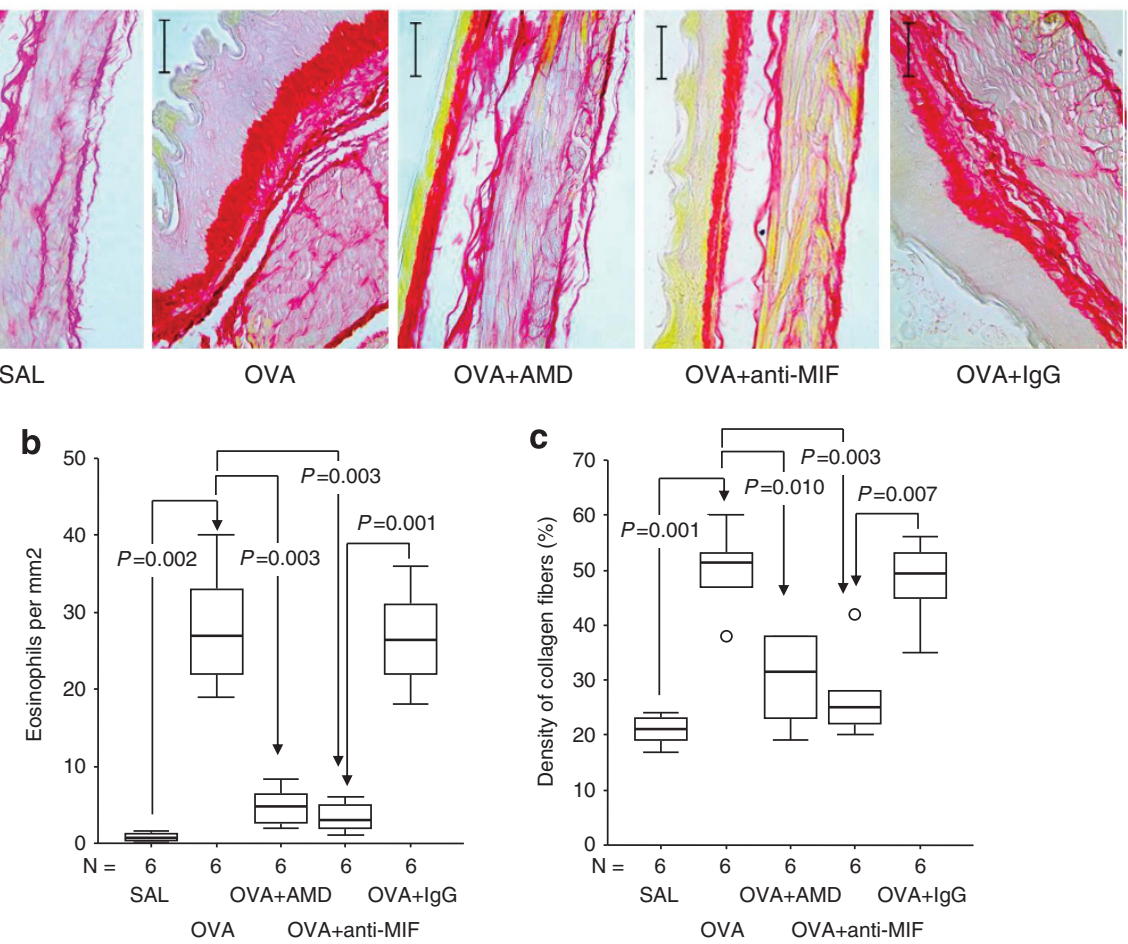

Figure 6 Macrophage migration inhibitory factor (MIF) antagonists abrogate full ovalbumin (OVA)-induced eosinophilic esophagitis. After OVA induction (OVA), animals presented significant morphological changes of Sirius red- and Picrosirius-stained esophageal sections characterized by intense cellular infiltration, predominantly of eosinophils and increased wall thickness, compared with saline-treated (SAL), and both OVA- and AMD3100- (OVA + AMD), and OVA- and anti-MIF antibody-treated animals (OVA + anti-MIF) (a). Analysis of esophageal sections revealed an increase in the number of eosinophils and the density of collagen fibers in OVA-treated animals compared with saline-treated, and with both OVA- and AMD or OVA- and anti-MIF-treated animals. An isotype-match immunoglobulin (IgG) was used as a control antibody (c). Horizontal bars represent medians, boxes represent the 25th and 75th percentiles, and vertical bars represent ranges of six animals/group. Length bars represent $20 \mu \mathrm{m}$. Differences were analyzed using ANOVA on ranks with Dunnett's test

We demonstrated that MIF expression concurs with the number of tissue eosinophils, and MIF was detected mostly within the cytosol of immune/inflammatory cells, predominantly defined as eosinophils by co-localization studies with confocal analysis. As previously reported, MIF actively participates in Th2-driven diseases, including asthma, ${ }^{15,19,22}$ atopic dermatitis, ${ }^{31}$ and parasitic infestations. ${ }^{16,20,21}$ Therefore, it has been postulated that the potent inflammatory actions of MIF in type 2 immune responses could be, at least in part, mediated by eosinophil recruitment and subsequent activation at sites of inflammation. ${ }^{16}$ In fact, little is known about MIF modulation in human eosinophils. Nevertheless, similar to our findings in EoE, increased MIF amounts have been shown in eosinophils in biopsy specimens of the nasal mucous membrane of rhinitis patients, ${ }^{32}$ and in bronchoalveolar lavage fluid from asthmatic patients. ${ }^{13}$ These results suggest the participation of MIF in airway inflammation and of eosinophils as a major source of MIF at inflammatory sites in atopic diseases. Taking together, the aforementioned findings combined with our demonstration of a remarkable role for MIF in eosinophil chemotaxis, and the detection of increased concentrations of MIF in supernatants of mucosal EoE esophageal explants, led us to hypothesize that MIF regulates eosinophil function in EoE. This effect is at least in part related to activation of CXCR4 receptor since the use of a selective antagonist abrogated MIF-induced eosinophil transmigration. Interestingly, CXCR4 is expressed on eosinophils and basophils, ${ }^{33,34}$ cells considered essential to the pathogenesis of EoE. ${ }^{26,35}$ Thus, it will be important to characterize the role of MIF and CXCR4 in basophil function and their specific role in the pathogenesis of EoE. 
a
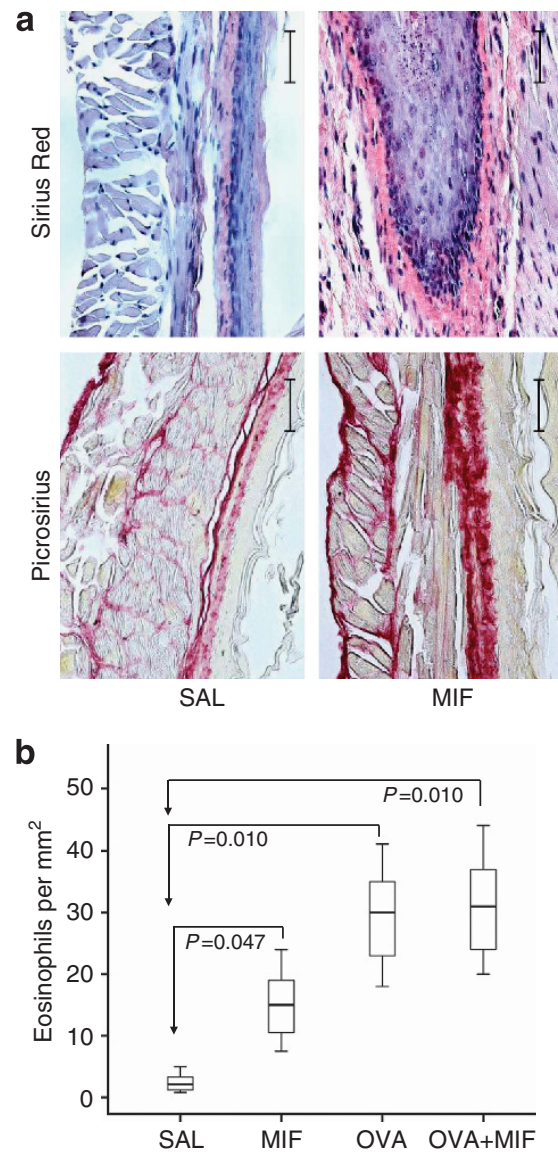

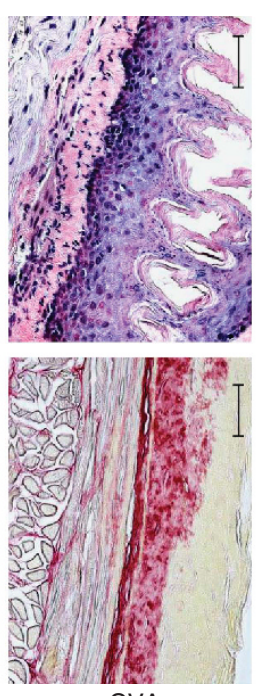

OVA
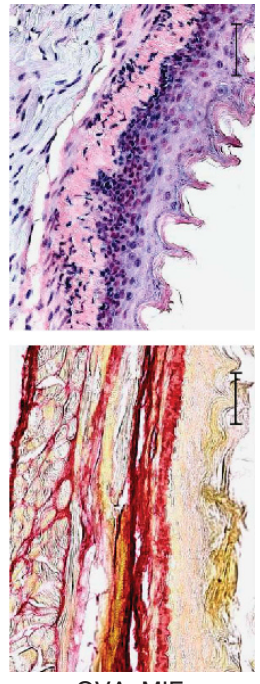

OVA+MIF

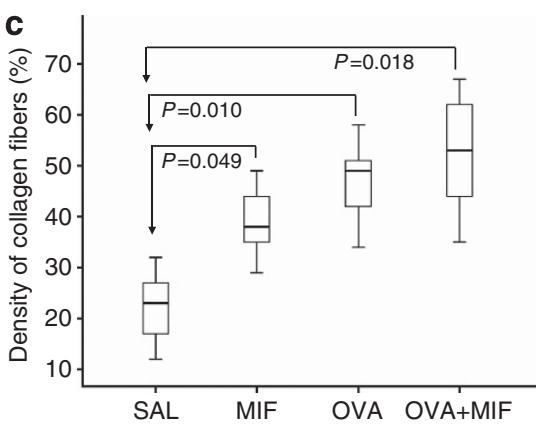

Figure 7 Recombinant macrophage migration inhibitory factor (MIF) induces eosinophilic esophagitis in ovalbumin (OVA)-sensitized mice. OVAsensitized C57BL/6 mice treated intranasally for 7 days with $0.5 \mu \mathrm{g}$ per mouse of recombinant mouse MIF (MIF), OVA (OVA), OVA and MIF (OVA + MIF), presented the typical morphological changes of Sirius red- and Picrosirius-stained esophageal sections characterized by intense cellular infiltration, predominantly of eosinophils and increased wall thickness compared with saline-treated animals (SAL) (a). Analysis of esophageal sections revealed an increase in the number of eosinophils (b) and the density of collagen fibers (c) in MIF, OVA, and OVA + MIF groups compared with saline-treated mice (SAL). Horizontal bars represent medians, boxes represent the 25th and 75th percentiles, and vertical bars represent ranges of six animals per group. Length bars represent $20 \mu \mathrm{m}$. Differences were analyzed using ANOVA on ranks with Dunnett's test.

Although MIF expression was remarkably increased in biopsy samples from EoE, levels were also higher in GERD relative to control patients. This finding appears to reflect the non-specific nature of MIF upregulation, which may be induced in various inflammatory conditions. ${ }^{11,36,37}$ However, given that EoE inflammation is orchestrated by a typical Th2 response, other mechanisms might be favoring MIF accumulation, in parallel with the eosinophil accumulation in the mucosa. It is possible that the milieu underlying EoE, based on a Th2 response, in contrast with the predominant Th1 response in GERD, ${ }^{38,39}$ may create a background in which eosinophils are attracted to and persist within the inflamed mucosa. In addition, eosinophil activation may induce the release of several inflammatory mediators by degranulation, ${ }^{40}$ probably also including MIF, which is compatible with our findings of MIF stored in cytosolic granules in EoE mucosal samples. From a mechanistic point of view, storing preformed inflammatory mediators such as MIF may represent an important biological strategy, especially for cells that are positioned at the interface between internal and external milieu and are expected to actively participate in rapid responses such as allergic and most innate immune reactions. Considering that in this study MIF was present mostly in eosinophils within the esophageal mucosa, it is conceivable that MIF may not constitute a primary trigger in EoE, but still emerges as a key element in the effector arm of EoE-associated inflammation. Because of the ability of eosinophils to produce MIF, ${ }^{13,17}$ it is also likely that effects observed in vivo might be a result of an autocrine/paracrine action of MIF released by eosinophil precursors in EoE.

Similar to previous studies demonstrating the existence of strong correlations between IL-5, IL-13, and eotaxin- $3,{ }^{27}$ and the key role of IL-13 in inducing eotaxin-3 in esophageal epithelial cells, ${ }^{7}$ we also have shown increased amounts of these type 2 cytokines and of eotaxin- 3 in the samples from patients with EoE. Nevertheless, in addition to the well-established Th2 background, these molecules apparently were not modulated by MIF, found in remarkably increased amounts in EoE samples. Although MIF has been shown to induce IL-5, IL-13, and eotaxin- 3 in the lungs of allergic mice and eotaxin- 3 by human eosinophils, ${ }^{15,17}$ we also observed that MIF constitutes a 
Table 1 Clinical and laboratory features of patients with eosinophilic esophagitis

\begin{tabular}{lccc}
\hline Diagnosis & EOE & GERD & Control \\
\hline Number of patients & 20 & 15 & $35.0(20-62)$ \\
Age in years: median (range) & $29.5(18-50)$ & 47 & $33.5(20-54)$ \\
Male gender (\%) & 75 & 27 & 50 \\
History of allergy (\%) & 60 & 60 & 0 \\
Previous PPI use (\%) & 40 & $4.0(0-8)$ & $2.5(0-6)$ \\
Blood eosinophilia: median (range) & $5.0(0-10)$ & $5(0-9)$ & 0 \\
Esophageal eosinophilia: median (range)
\end{tabular}

Abbreviations: EoE, eosinophilic esophagitis; GERD, gastro-esophageal reflux disease; history of allergy, including asthma, rhinitis, hay fever, or eczema; PPI, proton pump inhibitor; blood eosinophilia, cells per cubic milliliter; esophageal eosinophilia, cells per high-power field $\times 400$.

critical survival factor for eosinophils, regardless of IL-5 or IL13 levels. ${ }^{16}$ Therefore, in this study we hypothesize that MIF may act in a relative independence of IL-5, IL-13, or eotaxin-3, through its effects on eosinophil recruitment and survival, suggesting a role for MIF as a key component of both Th2 and innate immune response circuits involved in the control of inflammation in EoE.

In regard to the clinical parameters analyzed in this study, MIF amounts positively correlated to the number of eosinophils in tissue, and also to male gender. Interestingly, estrogen was previously shown to inhibit inflammation through the downregulation of local MIF production. ${ }^{41}$ In fact, anti-inflammatory effects of estrogen have been reported in males, ${ }^{42,43}$ and estrogen was shown to modulate the immune response both in vitro and in vivo. ${ }^{44}$ Because in this work we have demonstrated that MIF is a potent inducer of eosinophil infiltration, we thus speculate that MIF could also be a target of female sex steroids in the esophagus, potentially affecting mechanisms involved in the early phase of EoE, and also explaining, at least in part, the male predominance in EoE. To our knowledge, this information is new, and represents the first attempt to propose a mechanism for gender differences underlying EoE.

We used a mouse model of allergic EoE and demonstrated the resistance of $\mathrm{Mif}^{-/-}$to the development of the eosinophilic infiltration and collagen deposition even in the context of IL-13 overexpression. It was striking to observe the almost complete lack of inflammation and tissue remodeling in the absence of MIF. Others and we have previously demonstrated a critical role of MIF in mouse models of allergic asthma induced by OVA. ${ }^{15,19,45}$ In these experiments, the constitutive lack of MIF in $\mathrm{Mif}^{-/-}$mice would affect the priming and the challenge phase of the allergic response. However, our results here demonstrated that treatment with anti-MIF monoclonal antibody was also highly effective in preventing the signs of EoE. Similarly, treatment with a CXCR4 antagonist also protected against the signs of EoE, indicating that the axis MIF/CXCR4 is critical to EoE. At present, the contribution of SDF-1/CXCL12, the main ligand of CXCR4, on EoE is unknown and deserves investigation. The treatment protocols used in the present study were designed to block MIF/CXCR4 only in the challenge phase in mice that were previously immunized, indicating that MIF has an important role in the effector phase of the allergic response. Together, these results suggest that targeting MIF or blocking the CXCR4 receptor with AMD3100 (Plerixafor) might constitute a therapeutic option to treat patients with EoE. In fact, Plerixafor is currently approved by the FDA for short-term treatment while a recent clinical trial presented promising results in a long-term and low-dose treatment. ${ }^{46}$

In summary, these findings support the involvement of MIF in the pathogenesis of esophageal inflammation, and the mechanisms activated downstream of MIF are likely to maintain and even amplify the inflammatory response. The upregulation of esophageal MIF in the mucosa of patients with EoE and the protection from OVA-induced esophagitis in Mifdeficient mice are compatible with the participation of MIF in allergic inflammation and eosinophil accumulation in tissue. In fact, recombinant MIF promoted esophageal eosinophilia in allergic mice. Finally, these observations may implicate MIF signaling in the pathogenesis of EoE, and targeting MIF may constitute an innovative approach for future therapeutic interventions in human EoE.

\section{METHODS}

A description of antibodies, reagents, and treatments used in this study is presented as Supplementary Material.

\section{EoE patients and mucosal specimens}

Ethics statement. The study protocol was approved by the Ethical Committee of the University Hospital, Universidade Federal do Rio de Janeiro, and informed consent was obtained from all patients (approval number \# 111/08).

Study population and mucosal specimens. Consecutive patients regularly followed up at the outpatients unit for Esophageal diseases of the Division of Gastroenterology, at the University Hospital of the Universidade Federal do Rio de Janeiro, a tertiary referral center, were enrolled in this study. Esophageal specimens were obtained from inflamed mucosa of $20 \mathrm{EoE}$ and 15 GERD patients, and from macroscopically normal mucosa of 12 control individuals. In the context of endoscopically evident inflammation, biopsy specimens were obtained from the midpoint of the inflamed area in the middle and in the lower third, at least $2 \mathrm{~cm}$ above the gastro-esophageal junction. Additional biopsy specimens were obtained for routine histologic evaluation. 
The diagnosis of EoE was confirmed by clinical and endoscopic parameters, and defined histologically as having $>15$ eosinophils/ high-power field. ${ }^{2}$ In addition, other diseases were ruled out; and none of the EoE patients were on systemic or topical glucocorticoids, proton pump inhibitors, or dietary elimination at the time of their procedures. Patients with GERD had symptoms related to esophageal dysfunction and presenting with endoscopically evident erosive esophagitis, ${ }^{47}$ having $<15$ eosinophils/high-power field in either the distal or proximal biopsy specimens, were selected as an inflammatory control condition. None of the patients had endoscopic or histologic characteristics of Barrett's esophagus. Patients without esophageal disease, and submitted to diagnostic endoscopy because of non-specific upper gastrointestinal symptoms, not taking medications, who provided histologically normal tissue, constituted the control group. Table 1 describes clinical and laboratory parameters of the subjects enrolled in this study.

Histological analysis. Specimens were fixed in $40 \mathrm{gl}^{-1}$ formaldehyde saline, embedded in paraffin, cut into $5-\mu \mathrm{m}$ sections, stained with hematoxylin-eosin stain and Sirius red, and examined microscopically by two independent observers, unaware of the clinical or endoscopic diagnosis. Esophageal sections were studied according to recent consensus statements for histological assessment of EoE. ${ }^{2}$

Transwell migration assay. Eosinophils were isolated from EDTAanticoagulated blood samples of four healthy donors using an Eosinophil Isolation Kit (Millipore Corporation, Billerica, MA), resulting in $>96 \%$ purity and $>95 \%$ of cell viability. Eosinophil chemotaxis was determined using the QCM Chemotaxis Cell Migration Assay (Millipore Corporation) fitted with polycarbonate membrane of $5-\mu \mathrm{m}$ pore size. In a first set of experiments, purified eosinophils $\left(2 \times 10^{4}\right.$ cells per $\left.\mathrm{ml}\right)$ were incubated with RPMI-1640 medium supplemented with 5\% fetal calf serum (both from Gibco-Invitrogen, Carlsbad, CA), 2 mм L-glutamine, $50 \mu \mathrm{M} \mathrm{2-}$ mercaptoethanol, $10 \mathrm{~mm}$ HEPES, penicillin $\left(100,000 \mathrm{Ul}^{-1}\right)$ and streptomycin $\left(100 \mathrm{mgl}^{-1}\right)$ (Sigma Chemical, St Louis, MO) in the upper chambers, while rhMIF, rhEotaxin, or vehicle was diluted in $600 \mu \mathrm{l}$ of the culture medium and added to the lower chambers. To further confirm the effect of MIF on eosinophil chemotaxis, isolated eosinophils were incubated in the upper chambers, with CXCR4 antagonist (AMD3100), or the MIF antagonist ISO-1 diluted in culture medium, while MIF was added to the lower chambers. Cells were allowed to incubate at $37^{\circ} \mathrm{C}$ for $4 \mathrm{~h}$, after which cell density in the bottom chamber was determined by a colorimetric assay. Cells that migrated through the polycarbonate membrane were incubated with the cell stain solution of the kit, and then extracted for subsequent detection in a 96-well plate on a microplate reader, measuring the optical density at $560 \mathrm{~nm}$. Each in vitro experiment was performed in triplicate and repeated at least three times, with eosinophils purified from different donors.

Immunohistochemistry. Paraffin sections were cut onto slides pretreated with poly-lysine, and were used to characterize MIF-expressing cells, by the indirect immunoperoxidase technique. Briefly, deparaffinized sections were first incubated at $90{ }^{\circ} \mathrm{C}$ in $0.01 \mathrm{M}$ sodium citrate buffer (pH6.0) during $30 \mathrm{~min}$ for antigen retrieval. Then, slides were immersed in 3\% hydrogen peroxide in methanol for $10 \mathrm{~min}$ to block endogenous peroxidase activity. After being rinsed in phosphatebuffered saline (PBS) containing $0.5 \%$ Tween-20 for $10 \mathrm{~min}$, tissue sections were incubated with non-immune horse serum for $30 \mathrm{~min}$ and, subsequently, with the monoclonal antibody in a humidified chamber overnight, at $4{ }^{\circ} \mathrm{C}$. Immunohistochemical staining was carried out using an anti-MIF $(1: 1,000)$ antibody. Two sections from each sample were incubated with either PBS alone or mouse monoclonal IgG (concentration-matched) and served as negative controls. After being rinsed in PBS for $10 \mathrm{~min}$, all tissue sections were incubated for $30 \mathrm{~min}$ with the LSAB + system HRP kit (Dako, Glostrup, Denmark). Next, sections were rinsed in PBS and developed with a solution containing hydrogen peroxide and diaminobenzidine. Preparations were lightly counterstained in Harris's hematoxylin, dehydrated, and mounted in Permount (Fisher Scientific, Pittsburgh, PA).

Indirect Immunofluorescence and confocal laser scanning microscopy. In a double direct or indirect immunofluorescence study, sections were incubated overnight at $4{ }^{\circ} \mathrm{C}$ with $2.5 \%$ bovine serum albumin, $2.0 \%$ skimmed milk, $8.0 \%$ fetal bovine serum blocking buffer under shaking. Slides were rinsed once with PBS and $0.05 \%$ Tween-20 and then incubated with appropriately diluted primary antibodies in PBS. Tissue sections were incubated with anti-MIF antibody $(1: 1,000)$, and anti-CD3 FITC (1:50), or anti-CD68 FITC (1:50), or anti-major basic protein antibody (1:100), for $1 \mathrm{~h}$ at room temperature. After incubation, slides were rinsed three times and incubated with Alexa 488-conjugated anti-mouse IgG $(1: 1,000)$ or Alexa 633-conjugated anti-rabbit IgG $(1: 1,000)$, for $30 \mathrm{~min}$ at room temperature. Sections from each sample were incubated with either PBS alone or secondary antibody and served as negative isotype controls. Slides were air-dried, fixed for $5 \mathrm{~min}$ in a $1 \%$ paraformaldehyde solution, and mounted in an anti-fading medium containing 4',6-diamidino-2-phenylindole (DAPI) (Vector Labs, Burlingame, CA). Expression and localization of the proteins were observed with a Leica TCS-SP5 AOBS confocal laser-scanning microscope (Leica, Heidelberg, Germany), for capturing representative images of each sample.

Quantitative assessment of esophageal sections. Quantitative analysis of tissue sections (under light microscopy) was carried out using a computer-assisted image analyzer (Leica QWin Plus V 3.5.1, Leica Microsystems Ltd, Heerbrugg, Switzerland). Any epithelial and lamina propria cells exhibiting identifiable reactivity distinct from the background were regarded as positive. Percentages of MIF-positive cells were defined by the number of immunoreactive cells in relation to total cells (immunoreactive and non-immunoreactive cells; $\times 400$ magnification) in the epithelium per millimeter squared (counted in at least five different areas), of longitudinally sectioned esophageal mucosa. Two independent observers who were unaware of the experimental data examined all tissue sections and captured images.

In the double immunofluorescence studies with confocal microscopy analysis, co-localization was defined as the numbers of doublepositive cells (yellowish color) in relation to one of the single-positive cells (green color), in the epithelium per millimeter squared (counted in at least four different areas). Co-expression was further confirmed with confocal a laser scan microscopy co-localization tool. Two independent observers, who were unaware of the patients' data, examined all tissue sections and images captured.

RNA isolation and cDNA synthesis. Total RNA was obtained from fresh human and mice esophageal tissue, and isolation was performed using Absolutely RNA Miniprep Kit (Stratagene, La Jolla, CA). Equal amounts were then reverse transcribed using 1st Strand cDNA Synthesis Kit for RT-PCR (AMV) (Roche, Indianapolis, IN). The Nanodrop 2000 UV-vis Spectrophotometer (Thermo Scientific, Wilmington, DE) was used for quantifying and determining the RNA purity of samples. Primers specific for human MIF (forward, 5'-GTTCCTCTCCGAGCTCACCCAGCAGC- $3^{\prime}$; and reverse, $5^{\prime}$-GC AGCTTGCTGTAGGAGCGGTTCTG-3') were designed with Primer Express Software and synthesized by Eurofins MWG Operon (Huntsville, AL). The thermal cycle commenced with a hot start $94{ }^{\circ} \mathrm{C}$ for 5 min followed by 30 cycles each consisting of $94^{\circ} \mathrm{C}$ for $30 \mathrm{~s}$, annealing for $30 \mathrm{~s}$ at $56^{\circ} \mathrm{C}$, extension at $72{ }^{\circ} \mathrm{C}$ for $60 \mathrm{~s}$, and terminated after a final 7 -min period at $72{ }^{\circ} \mathrm{C}$. The products were run on a $1 \%$ agarose gel with SYBR Safe DNA gel stain 1:10,000 (Invitrogen, Eugene, OR) and glyceraldehyde phosphate dehydrogenase and $\beta$ actin were used as loading controls. For samples of the experimental EoE, we utilized primer sets from IDT (Integrated DNA Technologies, Coralville, IA) (Supplementary Table 1). 
Quantitative real-time PCR. To quantify the changes in mRNA levels, real-time RT-PCR was performed on the ABI Prism 7500 (Applied Biosystems, Foster City, CA) using RT2 Real Time SYBR Green/Rox PCR Master Mix (SABiosciences, Frederick, MD). For this purpose, levels of mRNA were normalized to the expression of glyceraldehyde phosphate dehydrogenase, $\beta$-actin and ribosomal protein L32 (RPL32), in human studies, or normalized to hypoxanthine phosphoribosyltransferase in the experimental model. For data analysis, the $\Delta \Delta \mathrm{Ct}$ method was used; determining the fold change for the target gene in each sample with 95\% confidence. Quantitative real-time PCR (qRT-PCR) for Mif gene was determined in triplicate. PCR cycles were performed according to the manufacturer's instructions.

Organ culture and cytokine measurements. Esophageal explants were cultured in RPMI-1640 medium supplemented with $10 \%$ fetal calf serum (Gibco-Invitrogen), $2 \mathrm{~mm}$ L-glutamine, $50 \mu \mathrm{M}$ 2-mercaptoethanol, $10 \mathrm{~mm}$ HEPES, penicillin $\left(100,000 \mathrm{Ul}^{-1}\right)$ and streptomycin $\left(100 \mathrm{mgl}^{-1}\right.$ ) (Sigma Chemical) for $24 \mathrm{~h}$ at $37^{\circ} \mathrm{C}$ in a $5 \% \mathrm{CO}_{2}$ humidified incubator. Samples were centrifuged and the supernatants used for measurement of the concentration of cytokines Eotaxin-3, IL5, and IL-13 (Peprotech, Rocky Hill, NJ), MIF (R\&D Systems, Minneapolis, MN), and IL-1 $\beta$ (eBiosciences, San Diego, CA) by commercial sensitive enzyme-linked immunosorbent assay (ELISA) method. The total protein content of the biopsy specimens was estimated by the Pierce BCA protein assay kit (Thermo Scientific, Rockford, IL), and used for normalizing the results. The minimum detectable concentration of human MIF, IL-1 $\beta$, IL-5, IL-13, and Eotaxin-3 was less than $5.0 \mathrm{pg} \mathrm{ml}^{-1}$.

Histomorphologic analysis of the esophagus. Specimens were fixed in $40 \mathrm{gl}^{-1}$ formaldehyde saline, embedded in paraffin, cut into $5-\mu \mathrm{m}$ sections, stained with hematoxylin-eosin stain, Sirius Red, and Picrosirius, and examined microscopically by two independent observers, to evaluate the number of eosinophils and the density of collagen fibers. Percentages of eosinophils were defined by the number of Sirius red-stained cells, carefully evaluated for morphology, in relation to total cells $(\times 400$ magnification $)$ in the epithelium per millimeter squared (counted in at least five different areas), of longitudinally sectioned esophagus.

For assessing the collagen deposition in the esophageal wall, the Picrosirius dye was used to stain collagen fibers in tissue, on serial paraffin sections. At least five different areas per tissue section were analyzed under light microscopy at an original magnification of $\times 100$. Density of collagen fibers was defined by the area positively stained for collagen in relation to total esophageal tissue. All histomorphologic analyses of tissue sections under light microscopy were performed using the computer-assisted image analyzer, as previously described.

\section{Experimental EoE and $\mathrm{Mif}^{-1-}$ mice}

Ethics statement for animal studies. The institutional animal care committee of the Health Sciences Centre of the Federal University of Rio de Janeiro approved the care and use of animals and procedures reported in this study (approval number \#IMPPG028), in accordance with the guidelines of the International Care and Use Committee of the National Institutes of Health, and Guide for the Care and Use of Laboratory Animals (National Research Council, the United States, 1996).

Animals. We utilized 6- to 8-week-old C57BL/6 and Mif $^{-1-}$ in a C57BL/ 6 background, as well as BALB/c mice. The $\mathrm{Mif}^{-/-}$mice were obtained as previously described ${ }^{20,48}$ and were backcrossed for 10 generations onto a C57BL/6 background. Mice were kept at constant temperature $\left(25^{\circ} \mathrm{C}\right)$ with free access to chow and water in a room with a 12-h light-dark cycle.

Induction of EoE and experimental design. Briefly, similar to previous studies using the OVA model of experimental EoE, ${ }^{49} \mathrm{WT}$ and $\mathrm{Mif}^{-1-}$ mice were sensitized by intraperitoneal injection of $50 \mu \mathrm{g}$ OVA and $1 \mathrm{mg}$ alum in $0.9 \%$ sterile saline on two occasions separated by 14 days. On day 15 , the mice were subsequently anesthetized and intranasally challenged with $150 \mu \mathrm{g}$ of OVA $(50 \mu \mathrm{l})$ on seven occasions over 10 days, followed by analysis performed $18 \mathrm{~h}$ after the last challenge. Following a similar protocol, groups of OVA-sensitized WT mice were challenge with OVA, recombinant mouse MIF ( $0.5 \mu \mathrm{g}$ per mouse), and the combination of OVA and MIF. In another set of experiments, OVA-sensitized $\mathrm{BALB} / \mathrm{c}$ mice were intraperitoneally treated with AMD3100 $\left(1.5 \mathrm{mg} \mathrm{kg}^{-1}\right)$, anti-MIF IgG1 monoclonal antibody, clone NIHIII.D9 ${ }^{50}\left(1 \mathrm{mg} \mathrm{kg}^{-1}\right)$, non-immune IgG1 isotype control (1 mg kg${ }^{1}$ ), or saline $1 \mathrm{~h}$ before each intranasal challenge. All mice were subsequently analyzed $18 \mathrm{~h}$ after the last intranasal challenge. Animals were euthanized by asphyxiation using an increasing concentration of $\mathrm{CO}_{2}$, and death was confirmed by cervical dislocation. The esophagus and lungs were then removed by thoracotomy. The esophagus was opened longitudinally and rinsed in saline several times before processing for histological assessment.

Statistical analysis. Statistical analysis was performed using the statistical software SPSS for Windows (Version 10.0.1, SPSS, Chicago, IL). Statistical differences among the experimental groups were evaluated with the Mann-Whitney or the Kruskal-Wallis ANOVA on ranks test in which multiple comparisons were carried out using the Dunnett's test, as appropriate. Values are expressed as medians with inter-quartile ranges. Correlation between the number of MIF-positive cells and eosinophils was assessed using the Spearman's rank correlation coefficient. The level of significance was set at $P<0.05$.

SUPPLEMENTARY MATERIAL is linked to the online version of the paper at http://www.nature.com/mi

\section{ACKNOWLEDGMENTS}

This work was supported by grants from the Brazilian Research Council (CNPq) and the FAPERJ (Fundação Carlos Chagas Filho de Amparo a Pesquisa do Estado do Rio de Janeiro). Drs Bucala and Leng are supported by AR050498. We acknowledge the technical assistance of Dr Monica Monerat and Grasiella Matioszek.

\section{DISCLOSURE}

The authors declared no conflict of interest.

c 2015 Society for Mucosal Immunology

\section{REFERENCES}

1. Rothenberg, M.E., Mishra, A., Collins, M.H. \& Putnam, P.E. Pathogenesis and clinical features of eosinophilic esophagitis. J. Allergy Clin. Immunol. 108, 891-894 (2001).

2. Liacouras, C.A. et al. Eosinophilic esophagitis: updated consensus recommendations for children and adults. J. Allergy Clin. Immunol. 128, 3-20. e26; 21-22 (2011)

3. Noel, R.J., Putnam, P.E. \& Rothenberg, M.E. Eosinophilic esophagitis. New Engl. J. Med. 351, 940-941 (2004).

4. Straumann, A. et al. Natural history of primary eosinophilic esophagitis: a follow-up of 30 adult patients for up to 11.5 years. Gastroenterology 125 , 1660-1669 (2003).

5. Mishra, A. \& Rothenberg, M.E. Intratracheal IL-13 induces eosinophilic esophagitis by an IL-5, eotaxin-1, and STAT6-dependent mechanism. Gastroenterology 125, 1419-1427 (2003).

6. Mishra, A., Schlotman, J., Wang, M. \& Rothenberg, M.E. Critical role for adaptive T cell immunity in experimental eosinophilic esophagitis in mice. J. Leukocyte Biol. 81, 916-924 (2007).

7. Blanchard, C. et al. IL-13 involvement in eosinophilic esophagitis: transcriptome analysis and reversibility with glucocorticoids. J. Allergy Clin. Immunol. 120, 1292-1300 (2007). 
8. Mishra, A., Hogan, S.P., Brandt, E.B. \& Rothenberg, M.E. IL-5 promotes eosinophil trafficking to the esophagus. J. Immunol. 168, 2464-2469 (2002).

9. Blanchard, C. et al. Eotaxin-3 and a uniquely conserved gene-expression profile in eosinophilic esophagitis. J. Clin. Investig. 116, 536-547 (2006).

10. Cavalcanti, M.G. et al. MIF participates in Toxoplasma gondii-induced pathology following oral infection. PLOS ONE 6, e25259 (2011).

11. Calandra, T. \& Roger, T. Macrophage migration inhibitory factor: a regulator of innate immunity. Nat. Rev. Immunol. 3, 791-800 (2003).

12. Assuncao-Miranda, I. et al. Contribution of macrophage migration inhibitory factor to the pathogenesis of dengue virus infection. FASEB J. 24, 218-228 (2010).

13. Rossi, A.G. et al. Human circulating eosinophils secrete macrophage migration inhibitory factor (MIF). Potential role in asthma. J. Clin. Investig. 101, 2869-2874 (1998).

14. Bacher, M. et al. An essential regulatory role for macrophage migration inhibitory factor in T-cell activation. Proc. Natl. Acad. Sci. USA 93, 78497854 (1996).

15. Magalhaes, E.S. et al. Macrophage migration inhibitory factor is essential for allergic asthma but not for Th2 differentiation. Eur. J. Immunol. 37, 1097-1106 (2007).

16. Magalhaes, E.S. et al. Macrophage migration inhibitory factor is critical to interleukin-5-driven eosinophilopoiesis and tissue eosinophilia triggered by Schistosoma mansoni infection. FASEB J. 23, 1262-1271 (2009).

17. Vieira-de-Abreu, A. et al. Cross-talk between macrophage migration inhibitory factor and eotaxin in allergic eosinophil activation forms leukotriene $\mathrm{C}(4)$-synthesizing lipid bodies. Am. J. Respir. Cell Mol. Biol. 44, 509-516 (2011).

18. Nakamaru, Y. et al. Macrophage migration inhibitory factor (MIF) contributes to the development of allergic rhinitis. Cytokine 31, 103-108 (2005).

19. Mizue, Y. et al. Role for macrophage migration inhibitory factor in asthma. Proc. Natl. Acad. Sci. USA 102, 14410-14415 (2005).

20. Rodriguez-Sosa, M. et al. Macrophage migration inhibitory factor plays a critical role in mediating protection against the helminth parasite Taenia crassiceps. Infect. Immun. 71, 1247-1254 (2003).

21. Stavitsky, A.B., Metz, C., Liu, S., Xianli, J. \& Bucala, R. Blockade of macrophage migration inhibitory factor (MIF) in Schistosoma japonicuminfected mice results in an increased adult worm burden and reduced fecundity. Parasite Immunol. 25, 369-374 (2003).

22. Yamaguchi, E. et al. Macrophage migration inhibitory factor (MIF) in bronchial asthma. Clin. Exp. Allergy 30, 1244-1249 (2000).

23. Bernhagen, J. et al. MIF is a noncognate ligand of CXC chemokine receptors in inflammatory and atherogenic cell recruitment. Nat. Med. 13, 587-596 (2007)

24. Vera, P.L., Iczkowski, K.A., Wang, X. \& Meyer-Siegler, K.L. Cyclophosphamide-induced cystitis increases bladder CXCR4 expression and CXCR4-macrophage migration inhibitory factor association. PLOS ONE 3 , e3898 (2008).

25. Lue, H., Dewor, M., Leng, L., Bucala, R. \& Bernhagen, J. Activation of the JNK signalling pathway by macrophage migration inhibitory factor (MIF) and dependence on CXCR4 and CD74. Cell. Signal. 23, 135-144 (2011).

26. Noti, M. et al. Thymic stromal lymphopoietin-elicited basophi responses promote eosinophilic esophagitis. Nat. Med. 19, 1005-1013 (2013).

27. Blanchard, C. et al. A striking local esophageal cytokine expression profile in eosinophilic esophagitis. J. Allergy Clin. Immunol. 127, 208-217. 217 e201-e207 (2011).

28. Franciosi, J.P., Tam, V., Liacouras, C.A. \& Spergel, J.M. A case-control study of sociodemographic and geographic characteristics of 335 children with eosinophilic esophagitis. Clin. Gastroenterol. Hepatol. 7, 415-419 (2009).
29. Chehade, M., Sampson, H.A., Morotti, R.A. \& Magid, M.S. Esophageal subepithelial fibrosis in children with eosinophilic esophagitis. J. Pediatr. Gastroenterol. Nutr. 45, 319-328 (2007).

30. Li-Kim-Moy, J.P., Tobias, V., Day, A.S., Leach, S. \& Lemberg, D.A. Esophageal subepithelial fibrosis and hyalinization are features of eosinophilic esophagitis. J. Pediatr. Gastroenterol. Nutr. 52, 147-153 (2011).

31. Shimizu, T. Role of macrophage migration inhibitory factor (MIF) in the skin. J. Dermatol. Sci. 37, 65-73 (2005).

32. Nakamaru, Y. et al. Macrophage migration inhibitory factor in allergic rhinitis: its identification in eosinophils at the site of inflammation. Ann. Otol. Rhinol. Laryngol. 113, 205-209 (2004).

33. Nagase, H. et al. Expression of CXCR4 in eosinophils: functional analyses and cytokine-mediated regulation. J. Immunol. 164, 5935-5943 (2000).

34. likura, M. et al. Chemokine receptors in human basophils: inducible expression of functional CXCR4. J. Leukocyte Biol. 70, 113-120 (2001).

35. Blanchard, C. \& Rothenberg, M.E. Basic pathogenesis of eosinophilic esophagitis. Gastrointest. Endosc. Clin. N. Am. 18, 133-143 (2008).

36. Lue, H., Kleemann, R., Calandra, T., Roger, T. \& Bernhagen, J. Macrophage migration inhibitory factor (MIF): mechanisms of action and role in disease. Microbes Infect. 4, 449-460 (2002).

37. Bozza, M.T., Martins, Y.C., Carneiro, L.A. \& Paiva, C.N. Macrophage migration inhibitory factor in protozoan infections. J. Parasitol. Res. 2012, 413052 (2012).

38. Fitzgerald, R.C. et al. Diversity in the oesophageal phenotypic response to gastro-oesophageal reflux: immunological determinants. Gut 50, 451-459 (2002).

39. Rieder, F. et al. Gastroesophageal reflux disease-associated esophagitis induces endogenous cytokine production leading to motor abnormalities. Gastroenterology 132, 154-165 (2007).

40. Mueller, S., Aigner, T., Neureiter, D. \& Stolte, M. Eosinophil infiltration and degranulation in oesophageal mucosa from adult patients with eosinophilic oesophagitis: a retrospective and comparative study on pathological biopsy. J. Clin. Pathol. 59, 1175-1180 (2006).

41. Ashcroft, G.S. et al. Estrogen modulates cutaneous wound healing by downregulating macrophage migration inhibitory factor. J. Clin. Investig. 111, 1309-1318 (2003).

42. Verdu, E.F., Deng, Y., Bercik, P. \& Collins, S.M. Modulatory effects of estrogen in two murine models of experimental colitis. American journal of physiology. Gastrointest. Liver Physiol. 283, G27-G36 (2002).

43. Aloisi, A.M. et al. Gender-related effects of chronic non-malignant pain and opioid therapy on plasma levels of macrophage migration inhibitory factor (MIF). Pain 115, 142-151 (2005).

44. Houdeau, E. et al. Sex steroid regulation of macrophage migration inhibitory factor in normal and inflamed colon in the female rat. Gastroenterology 132, 982-993 (2007).

45. Wang, B. etal. Cutting edge: Deficiency of macrophage migration inhibitory factor impairs murine airway allergic responses. J. Immunol. 177, 57795784 (2006).

46. McDermott, D.H. et al. A phase 1 clinical trial of long-term, low-dose treatment of WHIM syndrome with the CXCR4 antagonist plerixafor. Blood 123, 2308-2316 (2014).

47. Vakil, N. et al. The Montreal definition and classification of gastroesophageal reflux disease: a global evidence-based consensus. Am. J. Gastroenterol. 101, 1900-1920. 1943 (2006).

48. Bozza, M. et al. Targeted disruption of migration inhibitory factor gene reveals its critical role in sepsis. J. Exp. Med. 189, 341-346 (1999).

49. Cho, J.Y. et al. Smad3-deficient mice have reduced esophageal fibrosis and angiogenesis in a model of egg-induced eosinophilic esophagitis. J. Pediatr. Gastroenterol. Nutr. 59, 10-16 (2014).

50. Arjona, A. et al. Abrogation of macrophage migration inhibitory factor decreases West Nile virus lethality by limiting viral neuroinvasion. J. Clin. Investig. 117, 3059-3066 (2007). 